\title{
CARACTERISTICAS DA MORTALIDADE EM ADOLESCENTES BRASILEIROS DAS CAPITAIS DAS UNIDADES FEDERADAS
}

\begin{abstract}
YUNES, J. \& PRIMO, E. Características da mortalidade em adolescentes brasileiros das capitais das Unidades Federadas. Rev. Saúde públ., S. Paulo, 17:263-78, 1983.

KESUMO: São analisadas as características da mortalidade em adoles. centes nos grupos etários de 10-14, 15-19 e 10-19 anos, por sexo e grupo de causas, tanto para o Brasil e suas Regióes, como para as cidades de São Paulo e Recife (Brasil). Conclui-se que as áreas menos desenvolvidas apresentaram, quase sempre, coeficientes de mortalidade maiores que as mais desenvolvidas, e que as principais causas de óbito, nas áreas estudadas, são semelhantes às do Brasil, com variações na sua ordem de frequência. Observou-se, também, a ocorrência de maior risco para determinados grupos de causas, quando relacionados a grupo etário, sexo e regióes definidas. Chamou-se atenção para a necessidade de aumento de cobertura e melhoria da qualidade dos serviços de saúde destinados a esta população.
\end{abstract}

UNITERMOS: Adolescentes. Mortalidade. Serviço de saúde.

\section{INTRODUCAO}

A situação de saúde do adolescente deve ser considerada sob o enfoque de um processo vital contínuo, sobre o qual influem os fatores que atuaram nas fases anteriores do desenvolvimento biológico, tais como: herança genética, nutrição, estado prévio de saúde $e$ influência do meio ambiente físico, social e econômico sobre este estado de saúde. Por outro lado, as circunstâncias próprias dos meios social, cultural, geográfico e situação econômica, em que o adolescente está inserido, criam uma situação de risco contínus, a qual já está presente em etapas anteriores de seu ciclo vital. Por sua vez, a conduta do jovem contribui na determinação de seu estado de saúde, na de sua familia e na da comunidade, onde se conclui que as condições de saúde resultam do processo que é a vida e que o jovem amolda às condições de saúde da próxima geração.

A grande variedade de mudanças fisiológicas e psicossociais que ocorrem no adolescente fazem com que se considere este grupo como heterogêneo sob o ponto de vista dos problemas de saúde que apresentam, uma vez que estes problemas nem sempre se relacionam com um critério cronológico definido, mas sim de acordo com as etapas de maturação que se dão em períodos diferentes.

Como indicadores utilizados para se avaliar a situação de saúde do adolescente

* Do Departamento de Pediatria da Faculdade de Medicina da Universidade de Sáo Paulo Av. Dr. Arnaldo, 455 - 01246 - São Paulo, SP - Brasil.

* Bolsista do CNPq no Instituto da Criança do Hospital das Clínicas da Faculdade de Medicina da Universidade de São Paulo - Av. Dr. Enéas Carvalho de Aguiar, S/No - 05403 - São Paulo, SP - Brasil. 
YUNES. J. \& PRIMO, E. Características da mortalidade em adolescentes brasileiros das capitais das Unidades Federadas. Rev. Saúde públ., S. Paulo, 17:263-78, 1983.

tem-se, entre outros: as medidas antropométricas, morbidade, estado nutricional e mortalidade. Este último é que nos interessa mais de perto, ainda que não seja considerado um indicador positivo e embora reflita, indiretamente, o nivel de saúde da população em estudo.

O adolescente apresenta uma baixa mortalidade quando comparada a de outros grupos etários, porém, os coeficientes de mortalidade por causas mostram diferenças importantes, principalmente quando se comparam as regiões desenvolvidas com as subdesenvolvidas. Estas diferenças evidenciam a importância em serem reconhecidos os fatores predisponentes e condicionantes desta situação, bem como requerem uma clara definição intersetorial na assistência a este grupo.

A utilização deste indicador torna-se ainda mais importante quando se leva em conta que nos paises subdesenvolvidos a população é predominantemente jovem.

No Brasil, cerca de um quarto da população tem de 10 a 19 anos, o que corresponde, aproximadamente, a 27.500 .000 adolescentes em 1980. Destes, cerca de $13 \%$ correspondem à faixa etária de 10 a 14 anos e $11 \%$ na de 15 a 19 . Quanto à distribuição por sexo, $49,5 \%$ são do masculino e $50,5 \%$ do feminino para a faixa etária de 10 a 19 anos.

Este trabalho tem como principais objetivos:

a) estudar a mortalidade em adolescentes por grupo etário e sexo para o Brasil, suas regiões e as cidades de São Paulo e Recife;

b) estudar a mortalidade e madolescentes segundo grupo de causas, comparando-as por faixa etária e sexo para o Brasil e suas regiões e as cidades de São Paulo e Recife.

\section{METODOLOGIA}

Os dados de óbitos foram obtidos na Divisão Nacional de Epidemiologia do
Ministério da Saúde e os de população, no IBGE, para o ano de 1977.

Dadas as variações individuais con que st apresentam as mudanças que se produze'n entre as idades de 10 a 19 anos, é evidenta que os adolescentes formam um grupo heterogêneo sob o ponto de vista de saude. Assim, um desdobramento entre primeira adolescência (10 a 14 anos) e adolescência tardia (15 a 19 anos) é útil para fins estatísticos, dadas as diferenças, às vezes importantes, encontradas na análise comparativa, quer por idade e sexo, quer por causa de óbito.

Frente a diversificação das caracteristicas sócio-econômicas regrionais, tão evidentes no Brasil, um dos fatores que interferem nas principais causas de óbitos, apresentam-se os dados por regiōes. A inclusão das cidades de São Paulo e Recife permite a comparação entre centros de áreas metropolitanas localizadas em regiões que se opōem quanto às fases do desenvolvimento econômico em que se encontram.

A mortalidade por causa é, indubitavelmente, um indice útil para a ajudar avaliar as condiçōes de saúde do adolescente. No entanto, há de se ter em conta que a quantidade e a qualidade dos dados têm relação direta com a quantidade e integridade ou qualidade do atestado de óbito.

Em contrapartida, atestar um óbito corretamente depende da disponibilidade de médicos e de seu adequado preenchimento. Este problema representa, ainda hoje, uma das maiores limitaçóes para a existência de dados. Decorrente desta situação, ao se apresentar dados para o Brasil eles se referem somente às capitais das Unidades Federadas conforme listagem de causas de óbitos disponíveis no Ministério da Saude até julho de 1981. Portanto, sempre que se apresentarem tabelas especificando "Brasil" e "Regiōes", a situação refere-se às capitais.

A composição das Regiões Fisiográficas do Brasil por Capitais das Unidades Federadas é a seguinte: 
YUNES, J. \& PRIMO, E. Características da mortalidade em adolescentes brasileiros das capitais das Unidades Federadas. Rev. Saúde públ., S. Paulo, 17:263-78, 1983.

Norte (NO): Belém, São Luiz, Manaus, Rio Branco, Boa Vista e Macapá.

Nordeste (NE): Teresina, Fortaleza, Natal, João Pessoa, Recife, Maceió, Aracajú e Salvador.

Sudeste (SUD): Belo Horizonte, Vitória,
Niterói, Rio de Janeiro e São Paulo. Sul: Curitiba, Florianópolis e Porto Alegre. Centro-Oeste $(\mathrm{CO})$ : Cuibá, Goiânia, Brasilia e Porto Velho.

Para determinação do coeficiente de mortalidade de adolescentes, utilizou-se a seguinte relação:

Coeficiente de Mortalidade de

Adolescentes

No de óbitos de adolescentes

na faixa etária (10-19,10-14

ou 15-19 anos).

$$
=\text { População no respectivo grupo etário. }
$$

O coeficiente por causas é a razăo entre:

No de óbitos por determinada

Coeficiente de causa na faixa etária (10-19, Mortalidade 10-14 ou 15-19 anos). por causas

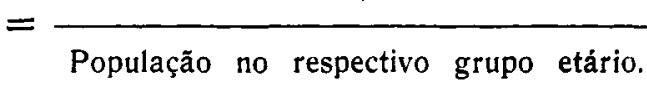
x 100.000 habitantes

Para uma análise mais pormenorizada das principais causas de óbitos do adolescente brasileiro, adotar-se-à a classificação da "Lista Brasileira para Mortalidade" proposta pclo Ministério da Saúde ${ }^{1}$, e que inclui, nos grupos de causas, as seguintes enfermidades:

\section{I - Doenças Infecciosas e Parasitárias}

- Doenças infecciosas intestinais

- Tuberculose

- Outras doenças bacterianas

- Doenças virais

- Rickettsioses e outras doenças transmitidas por artrópodes

- Doenças venéreas

- Outras doenças infecciosas e parasitárias e efeitos tardios de doenças infecciosas e parasitárias.

Il - Neoplasmas

- Neoplasmas malignos

- Neoplasmas benígnos, carcinoma "in situ", outros neoplasmas e os não-especificados.
III - Doenças das Glândulas Endócrinas, da Nutrição e do Metabolismo e Transtornos Imunitários

- Doenças endócrinas e metabólicas e transtornos imunitários

- Deficiências nutricionais

IV - Doenças do Sangue e dos Órgãos Hematopoéticos

V - Doenças do Sistema Nervoso e dos órgãos dos Sentidos

VI - Doenças do Aparelho Circulatório

- Febre reumática e doença reumática do coração

- Doença hipertensiva

- Doença isquêtmica do coração

- Doença da circulação pulmonar e outras formas de doença do coração.

- Doença cerebrovascular

- Outras doenças do aparelho circulatório

VII - Doenças do Aparelho Respiratório

- Doenças do trato respiratório superior 
YUNES, J. \& PRIMO, E. Características da mortalidade em adolescentes brasileiros das capitais das Unidades Federadas. Rev. Sauide públ., S. Paulo, 17:263-78, 1983.

- Outras doenças do trato respiratório

$$
\begin{aligned}
& \text { VIII - Doenças do Aparelho Digestivo } \\
& \text { IX - Doenças do Aparelho Geniturinári? } \\
& \text { - Doenças do aparelho urinário } \\
& \text { - Doenças dos órgãos genitais }
\end{aligned}
$$

$\mathrm{X}$ - Complicaçōes da Gravidez, do Parto e do Puerpério

- Aborto

- Causas obstétricas diretas

- Causas obstétricas indiretas

$\mathrm{XI}$ - Anomalias Congênitas

XII - Sintomas, Sinais e Afecçōes Mal Definidas

XIII - Causas externas

- Acidentes de transporte

- Envenenamento acidental e acidentes provocados em pacientes durante procedimento médico, reações anormais $\mathrm{e}$ complicaçōes tardias.

- Quedas acidentais

- Acidentes causados por fogo e chama

- Outros acidentes, incluindo efeitos tardios e efeitos adversos de drogas, medicamentos e substâncias biológicas usadas com finalidade terapêutica.

- Suicídios e lesões auto-infligidas

- Homicídios e lesões provocadas intencionalmente por outras pessoas

- Outras violências

Para efeito de análise, comparar-se-à a mortalidade de adolescentes do Brasil com os países que compõem as Regiōes da América do Norte e América do Sul.
Os paises que tinham dados mais próximos para esta análise foram:

- América do Norte: Bermudas, Canadá e Estados Unidos.

- América do Sul: Argentina, Colombia, Chile, Equador, Guiana Francesa (atual Suriname) Paraguai (área de informação), Perú, Uruguai e Venezuela.

\section{RESULTADOS E COMENTARIOS}

\section{1. - Mortalidade por Grupo Etário $e$ Sexo}

Não obstante a mortalidade em adolescentes seja considerada baixa, quando relacionada a outros grupos etários, observa-se, em geral, uma taxa mais elevada nos países subdesenvolvidos quando comparada com os desenvolvidos ". Para ilustrar esta afirmativa, verifica-se, conforme Tabela 1, que enquanto o coeficiente de mortalidade para o Brasil, no grupo etário 10 a 19 anos, é de $73,9(\mathrm{p} / 100.000$ habitantes $)$, na Suécia é de 46,0 .

Para os demais paises desenvolvidos, em geral, os valores apresentados são sempre menores do que os do Brasil. A situação é idêntica para os outros países subdesenvolvidos, onde a cifra está sempre acima de cem óbitos por 100.000 habitantes. Para os grupos etários restantes (10 a 14 e 15 a 19 anos), encontra-se, praticamente, 0 mesmo padrão anteriormente descrito.

Considerando a mortalidade em qualquer pais, nota-se também este comportamento, isto é, as regiões menos desenvolvidas apresentam, quase sempre, coeficientes de mortalidade maiores que os das mais desenvolvidas.

Para ilustrar este enunciado, toma-se o Brasil como exemplo. Ao serem comparadas as diversas regiões brasileiras, conforme se verifica na Tabela 2 , para os diversos grupos etários, a mortalidade nas Regiões Norte e Nordeste é, por via de regra, sempre maior que na Sul e Sudeste. 
YUNES, J. \& PRIMO, E. Características da mortalidade em adolescentes brasileiros das capitais das Unidades Federadas. Rev. Saúde públ., S. Paulo, 17:263-78, 1983.

T A B E L A 1

Coeficiente de mortalidade de adolescentes (por 100.600 habitantes) por grupos de idade em diversos países para anos próximos a 1977.

\begin{tabular}{lrcc}
\hline & \multicolumn{3}{c}{ Grupo etário } \\
\cline { 2 - 4 } & $10-19$ & $10-14$ & $15-\cdot 19$ \\
\hline Brasil * & 73,9 & 31,9 & 95.5 \\
Chile & 100,2 & $\ldots$ & $\ldots$ \\
México & 131,1 & 113.5 & 169.2 \\
Costa Rica & 66,1 & 46.8 & 90,4 \\
Tailândia & 157,9 & $\ldots$. & $\ldots 9.1 *$ \\
Estados Unidos ** & 75,9 & 33,7 & 106,0 \\
Austrália & 67,6 & 32,2 & 70,1 \\
Japão & 49,3 & 28,1 & 75,0 \\
Itália & 56,8 & 38,8 & 85,0 \\
Checoslováquia & 62,4 & 36,1 & 64,0 \\
Suécia & 46,0 & 27,7 & \\
& & & \\
\hline
\end{tabular}

Fontes: OMS 2 (1977).

* Brasil - dados brutos fornecidos pelo Ministério da Saúde.

* Estados Unidos - dados obtidos na Divisão de Estatística da Organização Panamericana da Saúde.

Ao se avaliar a mortalidade por faixa etária, comparando-se o grupo de 10 a 14 e de 15 a 19 anos, constata-se que para este último o coeficiente de mortalidade é sempre maior que para $o$ anterior e a mortalidade do grupo etário de 10 a 19 anos apresenta, sistematicamente, valores intermediários entre os dois grupos etários considerados. Este padrão de mortalidade pode ser verificado nas Tabelas 1 e 2 onde o coeficiente de mortalidade em adolescentes de 15 a 19 anos comparado ao grupo de 10 a 14 , geralmente está na proporção de dois para um.

Igualmente ass demais grupos etários, a diferenciação por sexo é padrão típico da mortalidade em adolescentes, sendo que a do sexo masculino é sempre maior que a do feminino.

Ao se comparar a mortalidade do adolescente por sexo para os diferentes grupos etários, corrobora-se não só que a mortalidade é sempre maior no sexo masculino, mas também que o grupo de mais alto risco é o de 15 a 19 anos.
Conforme se observa na Tabela 2, para - Brasil, a mortalidade de adolescentes de 10 a 14 anos para o sexo masculino é de 63,6 e para o feminino esta taxa é menor, ou seja, 40,6. No grupo etário de 15 a 19 anos, esta diferença ainda é maior, isto $\dot{e}$, o coeficiente de mortalidade no sexo masculino é de 128,5 enquanto que no feminino é de 67,3 óbitos por 100.000 habitantes. Para as regióes do Brasil verifica-se também comportamento semelhante, encontrando-se, porém, valores sempre maiores nas regiōes menos desenvolvidas. Faz exceção a mortalidade do grupo etário de 15 a 19 onde o coeficiente no sexo masculino em São Paulo é maior que o apresentado em Recife para o mesmo sexo. Este achado deve-se à diferença encontrada nas causas de óbito como se verificará adiante.

Ao se comparar o coeficiente de nortalidade do sexo masculino de 15 a 19 anos com o de 10 a 14 , depreende-se que para o primeiro grupo este coeficiente é, em geral, duas vezes igual ou maior que 0 apresentado pelo segundo. 
YUNES, J. \& PRIMO, E. Características da mortalidade em adolescentes brasileiros das capitais das Unidades Federadas. Rev. Saúde públ., S. Paulo, 17:263-78, 1983.

T A B E L A 2

Coeficiente de mortalidade de adolescentes (por 100.000 habitantes) por regiãn e capitais dos Estados de São Paulo e Pernambuco segunđo grupo etário e sexo - 1977.

\begin{tabular}{|c|c|c|c|c|c|c|c|c|c|}
\hline \multirow{3}{*}{ Região } & \multicolumn{9}{|c|}{ Grupo etário } \\
\hline & \multicolumn{3}{|c|}{$10-19$} & \multicolumn{3}{|c|}{$10-14$} & \multicolumn{3}{|c|}{$15-19$} \\
\hline & Masc. & Fem. & $\mathrm{T}$. & Masc. & Fem. & $\mathrm{T}$. & Masc. & Fem. & $T$ \\
\hline Brasil & 95.8 & 54.2 & 73.9 & 63.6 & 40.6 & 51,9 & 128.5 & 67.3 & 95.5 \\
\hline Norte & 155,3 & 92,9 & 122.6 & 110,4 & 59.0 & 84.2 & 208,7 & 128.7 & 165.3 \\
\hline Nordeste & 117,7 & 68.9 & 91,5 & 93,7 & 59,0 & 75,6 & 144,9 & 77.9 & 107.8 \\
\hline C. Oeste & 185,0 & 54.5 & 69,2 & 62.7 & 38.7 & 50,4 & 109.2 & 70.5 & 89.0 \\
\hline Sudeste & 75.7 & 39.1 & 56,7 & 41,8 & 26.8 & 34.2 & 112,6 & 51.1 & 79.8 \\
\hline Sul & 122,0 & 66.8 & 93,4 & 73,9 & 57,9 & 50,4 & 140.9 & 75,1 & 106.0 \\
\hline São Paulo & 107.2 & 55,1 & 80.4 & 56,2 & 33.8 & 44.9 & 162.8 & 76.3 & 117,1 \\
\hline Recife & 104,0 & 70,2 & 85.9 & 84,3 & 57,3 & 70,4 & 126.8 & 82,7 & 102,2 \\
\hline
\end{tabular}

Fonte: Dados brutos fornecidos pelo Ministério da Saúde.

\section{2. - Mortalidade Segundo Causas}

\subsection{1. - Por grupo etário}

Conforme afirmação anterior, para efeito de análise, comparar-se-à a mortalidade de adolescentes do Brasil com a de paises que compõem as Regiões da América do Norte e América do Sul (Tabela 3):"

Para a compreensão dos dados tanto do grupo etário de 10 a 14 anos como no de 15 a 19 chama a atenção a alta percentagem que representa na América do Sul e no Brasil a causa de óbito classificada como "outras enfermidades" en comparação com a América do Norte. Para esta última Região, no entanto, estas enfermidades representam cerca de $25 \%$ das causas no grupo etário de 10 a 14 anos e de 16,2\% no de 15 a 19 , enquanto que para a América do Sul e Brasil, a mortalidade por estas causas se duplicam para o grupo etário de 10 a 14 anos e se triplicam no de 15 a 19.

Esta situação traduz a grande dispersão de diagnóstico ou sua ausência nestas duas Regiões (América do Sul e Brasil), situaçāo que está associada as comentário inicial- mente feito, e por demais conlecidas, acerca d.) atestado de óbito.

No grupo etário de 10 a 14 anos, para as três Regiões que se está comparando, verifica-se que os acidentes e neoplasmas aparecem entre as cinco principais causas de óbito, variando somente a ordem de frequiencia. No grupo etário de 15 a 19 anos acresce-se como causa comum também para as três áreas as Doenças do Aparelho Circulatório.

No Brasil, tanto para o grupo etário ae 10 a 1 t. anos, como para o de 15 a 19 , as causas consideradas como Mal Definidas aparecem entre as cinco principais causas de óbito, refletindo, em parte, a precariedade da assistência médica, quer quanto a sua coberturá, quer quanto ao padrão ou qualidade de atendimento prestado.

Da mesma forma que para os grupos etários inferiores, as Doenças Infecciosas e Parasitárias também estão entre as cinc') principais causas de óbito no Brasil, traduzindo ainda um nivel bastante insatisfatório das condiçoes de saúde do adolescente. 
YUNES, J. \& PRIMO, E. Caracteristicas da mortalidade em adolescentes brasileiros das capitais das Unidades Federadas. Rev. Saúde puibl., S. Paulo, 17:263-78, 1983.

T A B E L A $\mathbf{3}$

Proporçăo (\%) de obitos de adolescentes segundo causas e grupo etário, na América do Norte e do Sul, 1975 e Brasil, 1977.

\begin{tabular}{|c|c|c|c|c|c|}
\hline \multirow{2}{*}{\multicolumn{2}{|c|}{ America do Norte }} & \multicolumn{2}{|c|}{$10-14$ anos } & \multirow{2}{*}{\multicolumn{2}{|c|}{ Brasil * }} \\
\hline & & América do & & & \\
\hline Causas & $\%$ & Causas & $\%$ & Causas & $\%$ \\
\hline Acidente & 53,4 & Acidente & 28,1 & Mal definidas & 17,8 \\
\hline Neoplasia & 12,0 & Pneumonia & 8,3 & Acidente $* *$ & 11,8 \\
\hline Anomalias & & & & Infecciosas e & \\
\hline Congenitas & 4,5 & Neoplasia & 5,8 & Parasitárias & 10,6 \\
\hline Pneumonia & 3,3 & Homlcidio & 4,6 & Aparelho & \\
\hline Aparelho & & & & Circulatório & 6,9 \\
\hline Circulatório & 2,7 & Tuberculose & 3,3 & Neoplasia & 6,9 \\
\hline Outras & 24,1 & Outras & 49,9 & Outras & 46,0 \\
\hline Total & 100 & \multicolumn{2}{|r|}{100} & & 100 \\
\hline \multirow{2}{*}{\multicolumn{2}{|c|}{ América do Norte }} & \multicolumn{2}{|c|}{$15-19$ anos } & \multirow{2}{*}{\multicolumn{2}{|c|}{ Brasil * }} \\
\hline & & América do & & & \\
\hline $\begin{array}{l}\text { Causas } \\
\text { Acldente }\end{array}$ & $\begin{array}{c}\% \\
59,6\end{array}$ & $\begin{array}{l}\text { Causas } \\
\text { Acldente }\end{array}$ & $\begin{array}{c}\% \\
32,7\end{array}$ & $\begin{array}{l}\text { Causas } \\
\text { Mal DefInldas }\end{array}$ & $\begin{array}{c}\% \\
13,5\end{array}$ \\
\hline Homiciálo & 8,7 & Homicidio & 5,7 & Acidente m* & 12,0 \\
\hline Suicidio & 7,7 & Neoplasia & 5,2 & Infecclosas e & \\
\hline Neoplasia & 5,9 & Aparelho & & Parasitária & 7,6 \\
\hline Aparelho & & Circulatório & 5,0 & Aparelho Circulat. & . 7,2 \\
\hline Circulatório & 1,9 & Suicldio & 5,0 & Neoplasia & 5,4 \\
\hline Outras & 16,2 & Outras & 46,4 & Outras & 54,3 \\
\hline Total & 100 & & 100 & & 100 \\
\hline
\end{tabular}

\subsection{2. - Por Grupo Etário e Sexo}

a - Mortalidade de 10 a 19 anos por sexo

Como se pode observar pela Tabela 4 , as cinco principais causas de óbito para 0 adolescente brasileiro de 10 a 19 anos são:

\section{- Causas Externas}

- Doenças Infecciosas e Parasitárias

- Doenças do Aparelho Circulatório

- Neoplasmas.

- Sintomas, Sinais e Atecções Mal Definidas

As Causas Externas apresentam um coeficiente de 34,3 óbitos por 100.000 habitantes, representando cerca de $45 \%$ do total de óbitos desta faixa etária. As demais causas apresentam um coeficiente bem menor, ou seja, variando de 0,6 (Doenças do Sangue e dos Órgãos Hematopoéticos) a 7,0 (Doenças Infecciosas e Parasitárias).

Ao se analisar as causas de óbitos por regiōes e capitais dos Estados de São Paulo e Pernambuco (Tabela 5) observa-se que as cinco principais causas para as Regiões Norte, Nordeste e Centro-Oeste são as mesmas que as do Brasil, variando somente, a sua ordem de freqüência. No entanto, para as Regiōes Sudeste e Sul e para as capitais de Pernambuco e São Paulo, as 
YUNES, J. \& PRIMO, E. Características da mortalidade em adolescentes brasileiros das capitais das Unidades Federadas. Rev. Saúde públ., S. Paulo, 17:263-78, 1983.

$$
\text { T A B E L A } \mathrm{A}^{\prime} 4
$$

Coeficiente de mortalidade de adolescentes de 10-19 anos (por 100.000 habitantes) por grupo de causas e sexo - Brasil, 1977.

\begin{tabular}{|c|c|c|c|}
\hline Grupo de Causas & Masculino & Feminino & Total \\
\hline Causas Externas & 53,1 & 17,3 & 34,3 \\
\hline Doenças Infecciosas e Parasitárias & 8,7 & 4,8 & 7,0 \\
\hline Doenças do Aparelho Circulatório & 5.8 & 5,3 & 5.5 \\
\hline Neoplasmas & 5.9 & 4,4 & 5.1 \\
\hline Mal Definidas & 4,5 & 4.1 & 4,3 \\
\hline $\begin{array}{l}\text { Doenças do Aparelho Respiratório } \\
\text { Complicacóes da Gravidez. Parto e }\end{array}$ & 4,9 & 3,9 & 4,1 \\
\hline Puerpério & 一 & 1,9 & 1,9 \\
\hline $\begin{array}{l}\text { Doenças do Sistema Nervoso e dos } \\
\text { orgåos dos Sentidos }\end{array}$ & 1,9 & 1,5 & 1,7 \\
\hline Doenças do Aparelho Digestivo & 1,5 & 0,9 & 1,2 \\
\hline Doenças do Aparelho Urinário & 1,0 & 1,1 & 1,1 \\
\hline $\begin{array}{l}\text { Anomalias Congẽnitas } \\
\text { Doenças das Glândulas Endocrinas, } \\
\text { da Nutrição e do Metabolismo a }\end{array}$ & 0,9 & 0,9 & 0,9 \\
\hline $\begin{array}{l}\text { Transtornos Imunitários } \\
\text { Doenças do Sangue e dos orgãos }\end{array}$ & 0,7 & 0.9 & 0,8 \\
\hline Hematopoéticos & 0,5 & 0.7 & 0,6 \\
\hline Todas as demais Doenças & 6,4 & 6,2 & 6.3 \\
\hline Total & 95.8 & 54,2 & 73,9 \\
\hline
\end{tabular}

Fonte: Dados brutos fornecidos pelo Ministério da Saúde.

Doenças do Aparelho Respiratório é que aparecem entre as cinco principais causas.

Para as Causas Externas, a Região que apresentou o maior coeficiente foi a Região Sul $(46,7 / 100.000)$. Em relação às Doenças Infecciosas e Parasitárias foi a Região Norte (23,5/100.000). O maior coeficiente para as Neoplasias ocorreu na Região Sul $(6,6 / 100.000$ e São Paulo $(7,0 / 100.000)$. As causas Mal Definidas apresentaram o maior coeficiente na Região Norte $(13,1 /$ 100.000) e as Doenças do Aparelho Respiratório na Região Sul $(7,7 / 100.000)$.

As cinco principais enfermidades que contribuíram como causas de óbito em adolescentes de 10 a 19 anos do sexo masculino foram, em ordem de freqüência, conforme Tabela 4, as seguintes:

\section{- Causas Externas}

- Doenças Infecciosas e Parasitárias

- Neoplasmas

- Doenças do Aparelho Circulatório

- Doenças do Aparelho Respiratório

As Causas Externas com um coeficiente de 53,1 óbitos por 100.000 habitantes foram responsáveis por mais da metade $(54 \%)$ do total de óbitos em adolescentes masculinos deste grupo etário. As demais causas contribuíram com uma proporção bem menor, variando 0 seu coeficiente de 0,5 (Doenças do Sangue e dos Orgãos Hematopoéticos) para 8,7 (Doenças Infecciosas e Parasitárias).

Ao se avaliar as causas do sexo masculino por região (Tabela 5), verificou-se que a Sudeste, Sul e São Paulo apresentaram as cinco principais causas de óbitos semelhantes às do Brasil, variando somente a 


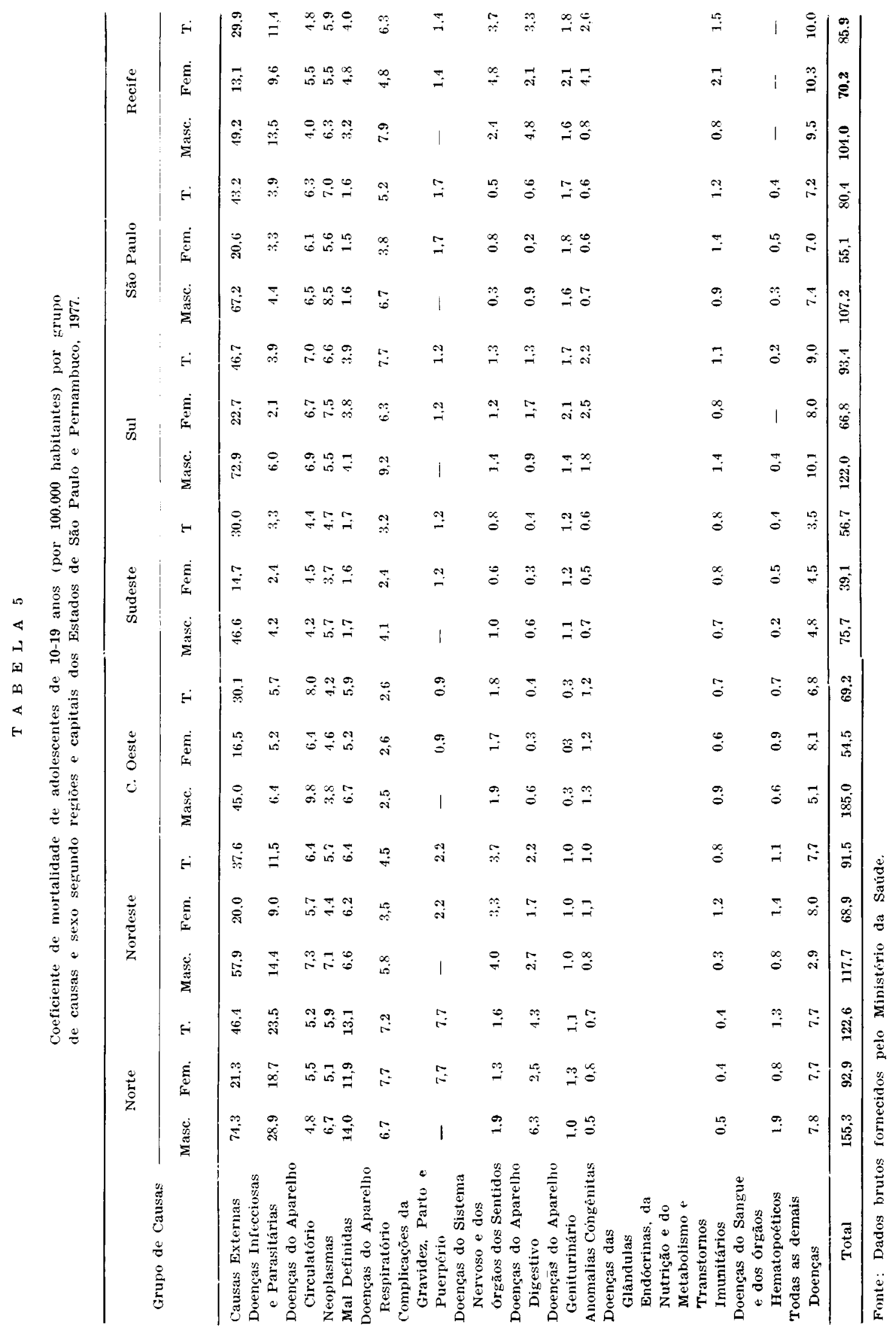


YUNES, J. \& PRIMO, E. Características da mortalidade em adolescentes brasileiros das capitais das Unidades Federadas. Rev. Saúde pübl., S. Paulo, 17:263-78, 1983.

T A E E L A 6

Coeficiente de mortalidade de adolescentes de 10-14 anos (por 100.000 habitantes) por grupo de causas e sexo - Brasil, 1977.

\begin{tabular}{|c|c|c|c|}
\hline \multirow{2}{*}{ Grupo de Causas } & \multicolumn{2}{|c|}{ Sexo } & \multirow{2}{*}{ Total } \\
\hline & Masculino & Feminino & \\
\hline Car'sas Externas & 30,5 & 12,0 & $\approx 1,1$ \\
\hline Doenças Infecciosas e Parasitárias & 7,5 & 5,0 & 6,2 \\
\hline Neoplasmas & 4.6 & 3,8 & 4,1 \\
\hline Doenças do Aparelho Respiratório & 4.7 & 3,6 & 4,1 \\
\hline Doenças do Aparelho Circulatório & 3.7 & 3.7 & 3,7 \\
\hline Mal Definidas & 2.6 & 3,0 & 2,8 \\
\hline Doenças do Sistema Nervoso e dos & & & \\
\hline órgãos dos Sentidos & 1.8 & 1.4 & 1,6 \\
\hline Doenças do Aparelho Digestivo & 0,9 & 1,0 & 0,9 \\
\hline Anomalias Congênitas & 1.0 & 0,9 & 0,9 \\
\hline $\begin{array}{l}\text { Doenças das Glândulas Endócrinas, } \\
\text { da Nutrição e do Metabolismo e } \\
\text { Transtornos Metabólicos }\end{array}$ & 0,5 & 0.9 & 0.7 \\
\hline Doenças do Aparelho Geniturinário & 0.5 & 0.9 & 0.7 \\
\hline Doenças do Sangue e dos órgãos & & & \\
\hline Hematopoéticos & 0,3 & 0.6 & 0,5 \\
\hline Complicações da Graviāez, Parto e & & & \\
\hline Puenperio & - & 0,1 & 4.2 \\
\hline Todas as demais Doenças & 4,8 & 3.7 & 0.7 \\
\hline Total & 63,6 & 40,6 & 51,9 \\
\hline
\end{tabular}

Fonte: Dados brutos fornecidos pelo Ministério da Saúde.

ordem de grandeza. Para as Regiões Norte, Nordeste e Centro-Oeste, as causas Mal Definidas é que se incluem entre as cinco principais.

Ainda en relação ao sexo masculino, o maior coeficiente indicado pelas Causas Externas, Doenças Infecciosas e Parasitárias e Mal Definidas ocorreu ná Região Norte, apontando, respectivamente, os seguintes valores: $74,3,28,9$ e $14,0 / 100.000$. As neoplasias apresentaram o maior coeficiente na Região Nordeste $(7,1 / 100.000)$ e São Paulo $(8,5 / 100.000)$; as Doenças do Aparelho Circulatório no Centro-Oeste (9,8/ 100.000) e as Doenças do Aparelho Respiratório no Sul $(9,2 / 100.000)$.

Para o sexo feminino, as cinco principais causas de óbito, em ordem decrescente de grandeza, para a faixa etária considerada (10 a 19 anos), foram:
- Causas Externas

- Doenças do Aparelho Circulatório

- Doenças Infecciosas e Parasitárias

- Neoplasmas

- Mal Definidas

Embora as Causas Externas continuem também contribuindo como a principal causa de óbito no sexo feminino $(17,3)$, na participaçăo proporcional no total de óbitos (31\%) é bem menor do que no do masculino (54\%). Depois das Causas Externas, o menor coeficiente $(0,7)$ também foi apresentado pelas Doenças do Sangue e dos órgãos Hematopoéticos e o maior $(4,8)$ pelas Doenças Infecciosas e Parasitárias. Esta última causa foi cerca de duas vezes maior no sexo masculino que no feminino, o que leva a supor que seja conseqüência 


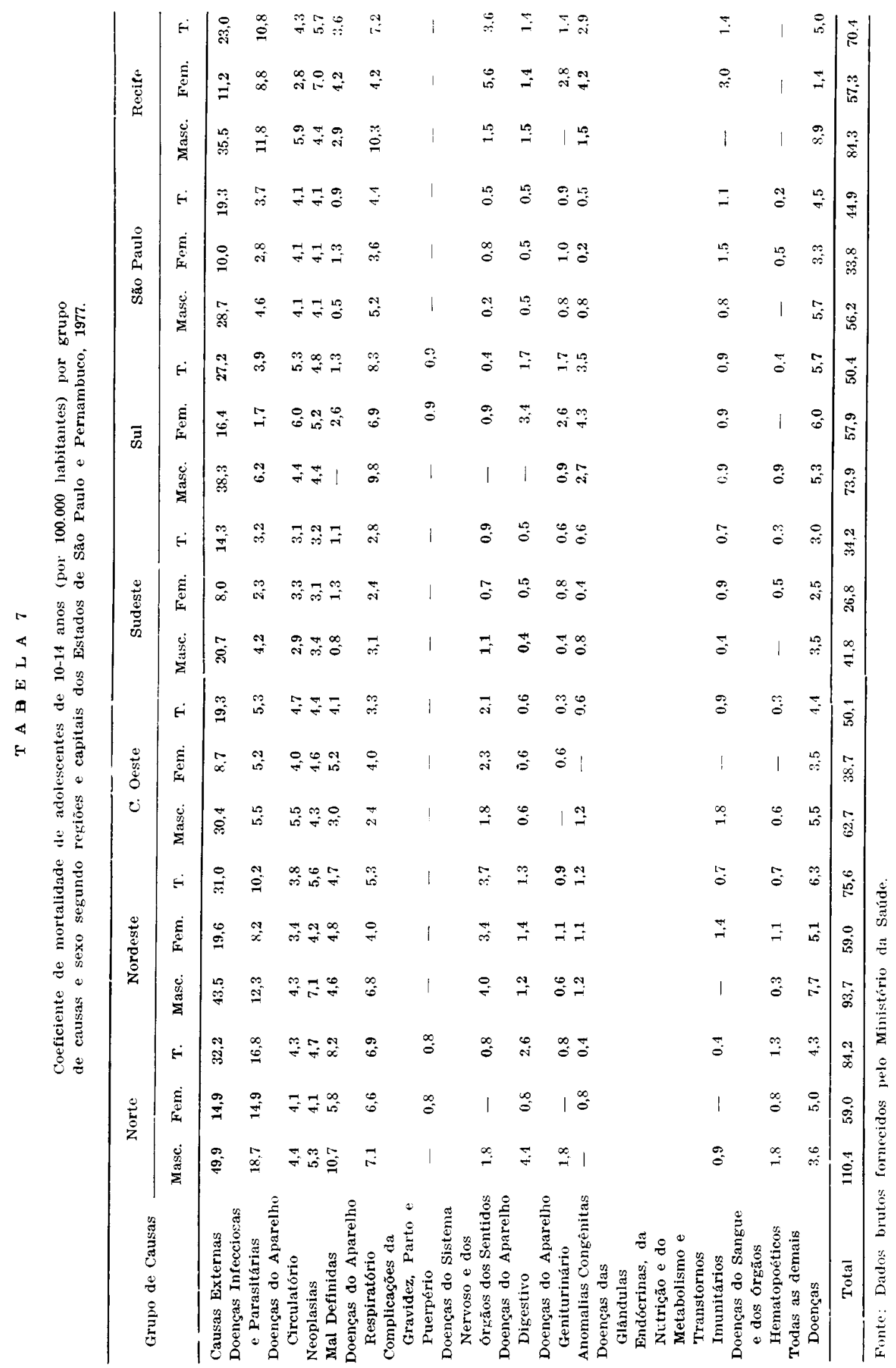


YUNES, J. \& PRIMO, E. Características da mo rtalidade em adolescentes brasileiros das capitais das Unidades Federadas, Rev. Saúde públ., S. Paulo, 17:263-78, 1983.

T A B E L A 8

Coeficiente de mortalidade de adolescentes de 15-19 anos (por 100.000 habitantes) por grupo de causa e sexo - Brasil, 1977.

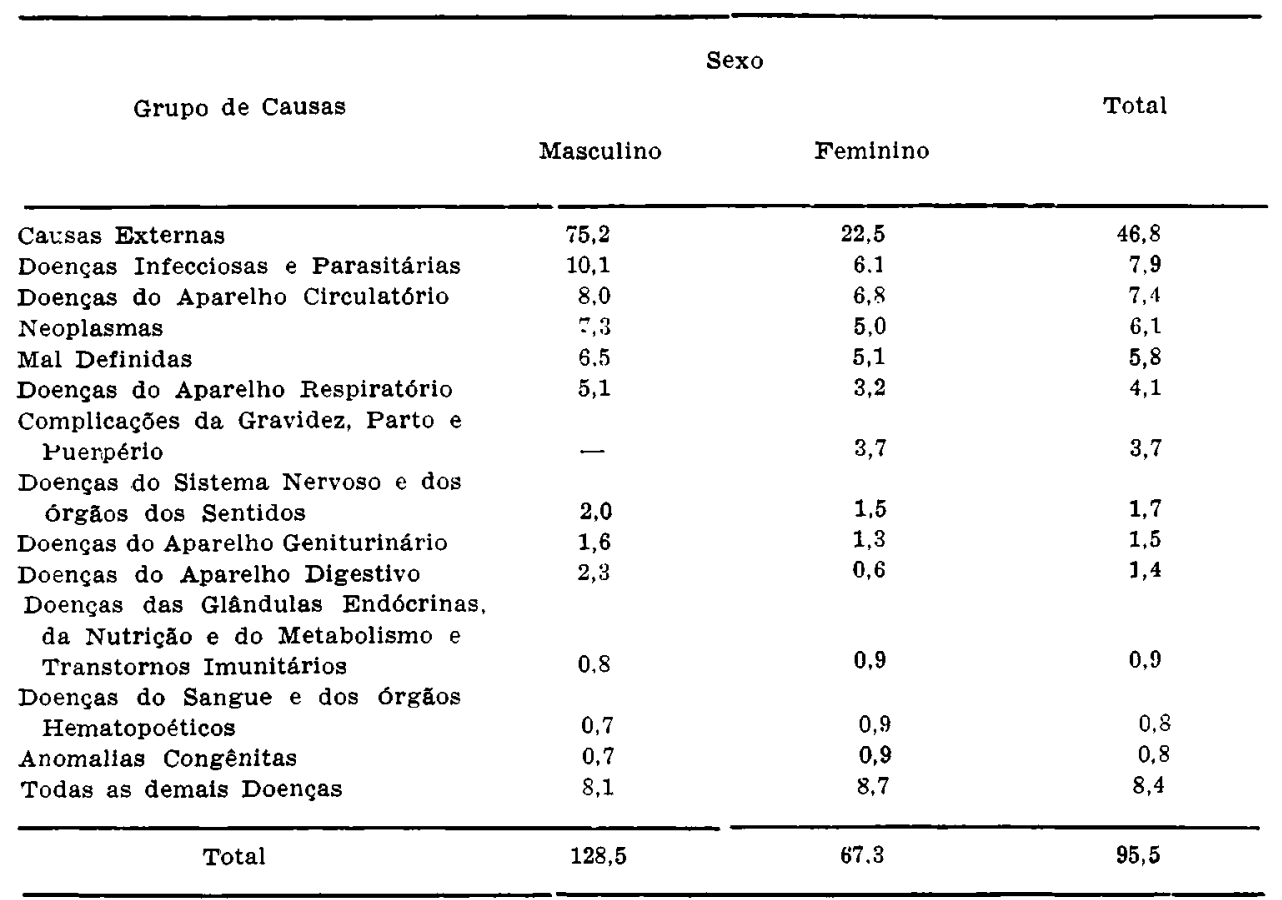

Fonte: Dados brutos fornecidos pelo Ministério da Saúde.

da maior exposição que o adolescente do sexo masculino apresenta em relação ao meio ambiente.

Comparando-se as causas do sexo feminino por Região (Tabela 5), corrobora-se que a Nordeste e Centro-Oeste apresentaram as cinco principais causas de óbitos semelhantes às do Brasil, variando, no entanto, sua ordem. Chama atenção que para a Região Norte as Complicações da Gravidez, Parto e Puerpério apareceram entre as cinco principais causas de óbito. Também as Doenças do Aparelho Respiratório apareceram entre as principais causas para as Regiōes Norte, Sudeste, Sul e São Paulo.

Ainda em relação ao sexo feminino, as Causas Externas, as Neoplasias e as Doenças do Aparelho Circulatório apresentaram os maiores coeficientes na Região Sul, com os respectivos valores de 22,$7 ; 7,5$ e $6,7 / 100.000$ habitantes. As mais altas taxas para as Doenças Infecciosas e Parasitárias; Mal Definidas e Complicações da Gravidez, Parto e Puerpério ocorreram na Região Norte com os respectivos coeficientes de 18,$7 ; 11,9 \mathrm{e}$ $7,7 / 100.000$.

b - Mortalidade de 10 a 14 anos por sexo

Ao serem avaliadas as principais causas de óbito em adolescentes brasileiros de 10 a 14 anos (Tabela 6 ), verifica-se que as 5 doenças que mais contribuiram no obituário, por ordem de freqüència, foram as seguintes:

\section{- Causas Externas}

- Doenças Infecciosas e Parasitárias

- Neoplasmas

- Doenças do Aparelho Respiratório

- Doenças do Aparelho Circulatório 


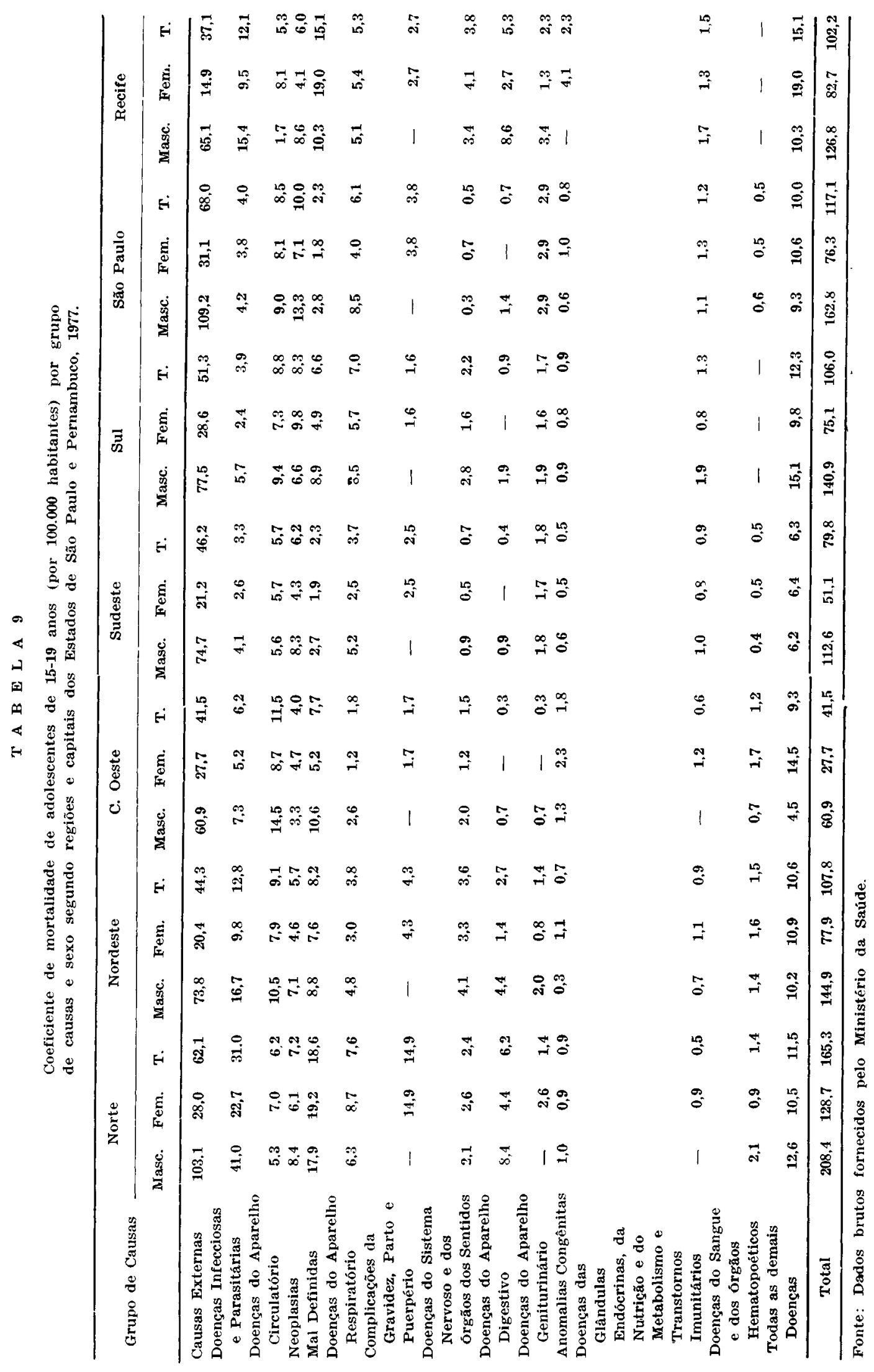


YUNES, J. \& PRIMO, E. Características da mortalidade em adolescentes brasileiros das capitals das Unidades Federadas. Rev. Saúde públ., S. Paulo, 17:263-78, 1983.

0 coeficiente de mortalidade por Causas Externas foi de 21,1 óbitos por 100.000 habitantes, o que representou cerca de $40 \%$ do total de óbitos desta faixa, apresentando, portanto, uma proporção menor do que no grupo etário de 10 a 19 anos.

Ao se comparar as causas de mortalidade por Região e Capitais dos Estados de São Paulo e Pernambuco, constatou-se que as cinco principais causas para as Regiōes Sudeste, Sul, São Paulo e Recife ,são as mesmas que as do Brasil, variando somente a ordem de frequiència destas. Para as demais Regiōes (Norte, Nordeste e Centro-Oeste), apareceram entre as cinco principais causas as Mal Definidas, conforme se observa na Tabela 7 .

As causas Externas, as Doenças Infecciosas e Parasitárias e as Mal Definidas apresentaram os seus maiores coeficientes na Região Norte e os valores destas taxas foram, respectivamente, $32,2,16,8$ e $8,2 /$ 1100.000 . Em relação às Neoplasias, os seus maiores coeficientes ocorreram na Região. Nordeste $(5,6 / 100.000)$ e Recife $(5,7 /$ /100.000).

As doenças do Aparelho Respiratório e Circulatório tiveram suas maiores taxas na Região Sul, onde os coeficientes foram, respectivamente, de 8,3 e 5,3/100.000 habitantes.

Ao serem comparadas as causas de óbito neste grupo etário, por sexo, conforme Tabela 6, observou-se que as cinco principais doenças que contribuiram para a mortalidade seguiram a mesma ordem de freqüência vista anteriormente. Também aqui a mortalidade por Causas Externas foi maior no sexo masculino $(30,5)$ que no feminino $(12 / 100.000)$.

Ao serem analisadas as causas de mortalidade por sexo, nas diferentes Regiōes $\mathrm{e}$ Capitais dos Estados de São Paulo e Pernambuco, demonstrou-se que as cinco principais causas de óbito seguem a mesma ordem de frequêencia que a verificada para - Brasil, com uma única diferença: na Região Sul, as Anomalias Congênitas apa- receram como a quinta principal causa no sexo feminino. (Tabela 7).

Para o sexo masculino, o maior coeficiente apresentado pelas Causas Externas e Drenças Infecciosas e Parasitárias ocorreram na Região Norte onde as taxas foram, respectivamente, de 49,9 e 18,7/ 1100.000 . O maior coeficiente por Neoplasias foi no Nordeste $(7,1 / 100.000)$.As Doenças do Aparelho Respiratório mostraram as maiores taxas na Região Sul $(9,8 / 100.000)$ e em Recife $(10,3 / 100.000)$, enquanto as do Aparelho Circulatório na Regiāo Centro-Oeste $(5,5 / 100.000)$ e também Recife $(5,9 / 100.000)$.

Em relação ao sexo feminino os maiores coeficientes apresentados pelas Causas Éxternas ocorreram na Região Nordeste $(19,6 / 100.000)$. A maior taxa de Doenças Infecciosas e Parasitárias deu-se na Região Norte $(14,9 / 100.000)$, ao passo que as Neoplasias, as Doenças do Aparelho Respiratório e as Doenças do Aparelho Circulatório tiveram seus maiores coeficientes na Região Sul, apontando respectivamente os seguintes valores: 5,2; 6,9 e 6,0/100.000. Também, em Recife, constatou-se um coeficiente elevado de mortalidade por Neoplasias $(7,0 / 100.000)$.

\section{c - Mortalidade de 15 a 19 anos por sexo}

Para o grupo etário de 15 a 19 anos, conforme se về na Tabela 8 , as cinco principais causas de óbito do adolescente brasileiro são:

\section{- Causas Externas}

- Doenças Infecciosas e Parasitárias

- Doenças do Aparelho Circulatório

- Neoplasias

- Sintomas, Sinais e Afeç̧ões Mal Definidas

Neste grupo etário, não só as Causas Externas foram a principal causa da morte (46,8 óbitos por 100.000 habitantes), como também, elas representaram o maior peso na mortalidade do adolescente $(49 \%)$ quando comparado com o da faixa etária de 10 a 14 anos (40\%). 
YUNES, J. \& PRIMO, E. Características da mortalidade em adolescentes brasileiros das capitais das Unidades Federadas, Rev. Saude puibl., S. Paulo. 17:263-78, 1983.

Ao se comparar as principais causas por Região (Tabela 9), comprovou-se que a Nordeste e a Centro-Oeste se assemelham a do Brasil, variando somente a ordem de frequiência. Para as Regiōes Sudeste e Sul e cidade de São Paulo, as Doenças do Aparelho Respiratório são as que se incluem entre as cinco principais causas de óbito. Chama atenção também que, para a Região Norte, as Complicaçóes da Gravidez, Parto e Puerperio são as que estão entre as cinco principais causas de mortalidade.

As Causas Externas, Doenças Infecciosas e Parasitárias, as Mal Definidas e as Complicaçōes da Gravidez, Parto e Puerpério são as que apresentaram os maiores coeficientes na Região Norte, sendo, respectivamente, de: 62,$1 ; 31,0 ; 18,6$ e 14,9 óbitos por 100.000 habitantes.

O maior coeficiente apresentado pelas Doenças do Aparelho Circulatório foi na Região Centro-Oeste $(11,5 / 100.000)$ e pelas Neoplasias na Região Sul $(8,3 / 100.000)$ e São Paulo (10,0/100.000).

Ao se analisar as cinco principais causas de óbito por sexo (Tabela 8), notou-se que ela segue, em geral, a mesma ordem de frequiencia que para o Brasil como um todo.

Chama atenção também, que no grupo etário considerado (15 a 19 anos) a mortalidade por causas externas no sexo masculino $(75,2$ óbitos por 100.000 habitantes) foi três vezes maior que na do sexo feminino $(22,5)$. Portanto, o grupo de adolescentes que apresenta o maior risco de morrer por Causas Externas são os de sexo masculino na faixa etária de 15 a 19 anos.

Ao serem comparadas as outras causas de óbito por sexo no grupo etário considerado, depreendeu-se que elas também são maiores no sexo masculino mas em proporções bem menores.

As cinco principais causas de óbitos nos sexos masculino e feminino no Nordeste, Centro-Oeste e Sul são semelhantes às anteriormente referidas para 0 adolescente brasileiro como um todo, variando somente a ordem de grandeza (Tabela 9). Para o sexo masculino, aparece entre as cinco principais causas de óbito as Doenças do Aparelho Digestivo na Região Norte e em Recife.

Quanto ao sexo feminino, chama a atenção que as complicações da Gravidez, Parto e Puerpério apareceram entre as cinco principais causas de morte na Regrão Norte, Sudeste e São Paulo.

Para o sexo masculino, conforme Tabela 9, o maior coeficiente de mortalidade por Causas Externas ocorreram na Região Norte $(103,1 / 100.000)$ e São Paulo (109,2/ 100.000). Também as Doenças Infecciosas e Parasitárias e as causas Mal Definidas tiveram a maior taxa na Região Norte, sendo, respectivamente, de 41,0/100.000 e $17,9 / 100.000$. No que se refere às Doenças do Aparelho Circulatório, o maior coeficiente apresentado foi na Região Centro-Oeste com 14,5 óbitos por 100.000 habitantes. As Neoplasias tiveram a maior taxa na Região Norte $(8,4 / 100.000)$ e em São Paulo (13/100.000). Quanto às Doenças do Aparelho Respiratório foi na Região Sul e em São Paulo onde os coeficientes foram maiores (8,5/100.000).

Em relação ao sexo feminino, as Doenças Infecciosas e Parasitárias, as Mal Definidas, as Complicaçōes da Gravidez, Parto e Puerpério e as Doenças Respiratórias tiveram os seus maiores coeficientes na Regiāo Norte, tendo as suas taxas, respectivamente, os seguintes valores: $22,7,19,2,14,9$ e 8,7 / 100.000.

Para este mesmo sexo, os maiores coeficientes para as Causas Externas deram-se na Região Sul $(28,6 / 100.000)$ e São Paulo (31,1/100.000). As Doenças do Aparelho Circulatório e as Neoplasias apresentaram as maiores taxas, respectivamente, na Região Centro-Oeste $(8,7 / 100.000)$ e na Sul $(9,8 /$ $100.000)$.

O padrão de mortalidade encontrado no adolescente, requer, para que haja uma contribuição na melhoria do seu nivel de saúde, que se ampliem os programas e atividades de saúde destinados a esta população e que eles devam ser multidi- 
mensionais, amplamente acessiveis e integrais, devendo considerar, portanto, também os fatores de ordem econômica, social, ambiental e cultural. Para isto deve se considerar o adolescente tanto como objeto das atividades dos programas, na qualidade de usuário, como sujeito em seu papel potencial de promotor das atividades de saúde correspondente ${ }^{4}$.

\section{CONCLUSÖES}

1. O maior risco de mortalidade em adolescentes foi no sexo masculino, na faixa etária de 15 a 19 anos e nas regiōes menos desenvolvidas.

2. As principais causas de óbito foram: Causas Externas; Doenças Infecciosas e Parasitárias; Doenças đo Aparelho Circulatório; Neoplasias; Causas Mal Definidas e Doenças do Aparelho Respiratório. Estas causas variaram na sua ordem de freqüiencia segundo idade, sexo e áreas comparadas.

3. As Causas Externas no sexo masculino; as Doenças Infecciosas e Parasitárias; as Causas Mal Definidas para ambos os sexos e as Complicações da Gravidez, Parto e Puerpério apresentaram os maiores coeficientes de mortalidade na Região Norte.

4. As Doenças do Aparelho Respiratório, para o sexo masculino; as Anomalias Congênitas, as Causas Externas $\mathrm{e}$ as Doenças do Aparelho Respiratório para o sexo feminino apresentaram as maiores taxas de mortalidade na Região Sul.

5. As Doenças do Aparelho Circulatório para o sexo masculino apresentaram os coeficientes mais elevados de mortalidade na Região Centro-Oeste.

6. A presença das Doenças Infecciosas e Parasitárias, Sintomas, Sinais e Afecções Mal Definidas e as Complicações da Gravidez, Parto e Puerpério, entre as principais causas de óbito, além de traduzirem um nível insatisfatório das condições de saúde do adolescente brasileiro, refletem, em parte, a precaridade da assistência médica para este grupo considerado.

YUNES, J. \& PRIMO, E. [Characteristics of Brazilian adolescent mortality]. Rev. Saúde públ., S. Paulo, 17:263-78, 1983.

ABSTRACT: The characteristics of adolescent mortality in the age group, sex and region was observed. Attention is called to the necessity of Brazil as whole and its constituint regions and for the cities of $S$. Paulo and Recife specifically are analyzed. It is concluded that the adolescent mortality rate was almost always bigger in the underdeveloped areas than in the developed ones. The main causes of death in the areas studied are similar to those in Brazil, varying only their order of frequency. The occurence of higher risk from particular causes of death according to age group, sex and region was observed. Attention is called to the necessity of improving the quantity and quality of health services to the adolescent population.

UNITERMS: Adolescence. Mortality. Health services.

\section{REFERENCIAS BIBLIOGRÁFICAS}

1. MINISTERIO DA SAƯDE. Divisão Nacional de Epidemiologia. Lista brasileira para mortalidade. Brasília, 1980.

2. ORGANIZACION MUNDIAL DE LA SALUD. Comite de Expertos en Necesidades de Salud de los Adolescentes, Ginebra, 1976. Informe, Ginebra, 1977. (Ser. Inf. técn.. 609 ).

3. ORGANIZACION PANAMERICANA DE LA SALUD. Condiciones de salud del niño en las Americas. Washington, D.C., 1979. (Publ., cient., 381).

4. ROSSELOT VICUÑA, J. La salud del adolescente $y$ del jovem en America Latina $y$ el Caribe. Bol. Ofic. sanit. panamer., 83:295-309, 1977. 